\title{
A field-based modeling study on ecological characterization of hourly host-seeking behavior and its associated climatic variables in Aedes albopictus
}

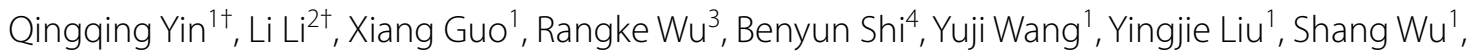
Yicheng Pan' ${ }^{1}$, Qi Wang ${ }^{1}$, Tian Xie', Tian Hu' ${ }^{1}$, Dan Xia ${ }^{1}$, Shang Xia ${ }^{5}$, Dzinkambani Moffat Kambalame', Wanyu Li ${ }^{1}$, Zhangyao Song ${ }^{1}$, Siyun Zhou ${ }^{1}$, Ye Deng ${ }^{1}$, Yu Xie $^{1}$, Xiao-Nong Zhou ${ }^{5}$, Chunmei Wang ${ }^{1 *}$, Xiao-Guang Chen ${ }^{1}$ and Xiaohong Zhou ${ }^{*}$

\begin{abstract}
Background: The global spread of mosquito-borne diseases (MBD) has presented increasing challenges to public health. The transmission of MBD is mainly attributable to the biting behaviors of female mosquitoes. However, the ecological pattern of hourly host-seeking behavior in Aedes albopictus and its association with climatic variables are still not well understood, especially for a precise requirement for establishing an effective risk prediction system of MBD transmission.

Methods: Mosquito samples and data on mosquito hourly density and site-specific climatic variables, including temperature, relative humidity, illuminance and wind speed, were collected simultaneously in urban outdoor environments in Guangzhou during 2016-2018. Kernel regression models were used to assess the temporal patterns of hourly host-seeking behavior in mosquito populations, and negative binomial regression models in the Bayesian framework were used to investigate the associations of host-seeking behavior with climatic variables.

Results: Aedes albopictus was abundant, constituting 82\% (5569/6790) of the total collected mosquitoes. Host-seeking behavior in Ae. albopictus varied across time and was significantly influenced by climatic variables. The predicted hourly mosquito densities showed non-linear relationships with temperature and illuminance, whereas density increased with relative humidity but generally decreased with wind speed. The range of temperature estimates for female biting was $16.4-37.1^{\circ} \mathrm{C}$, peaking at $26.5^{\circ} \mathrm{C}$ (95\% credible interval: $\left.25.3-28.1\right)$. During the favorable periods, biting behavior of female Ae. albopictus was estimated to occur frequently all day long, presenting a bimodal distribution with peaks within 2-3 h around both dawn and dusk (05:00-08:00 h and 16:00-19:00 h). Moreover, a short-term association in hourly density between the females and males was found.
\end{abstract}

Conclusions: Our field-based modeling study reveals that hourly host-seeking behavior of Ae. albopictus exhibits a complex pattern, with hourly variation constrained significantly by climatic variables. These findings lay a foundation

\footnotetext{
*Correspondence: wangcmq@163.com; daizhouxh@163.com

${ }^{\dagger}$ Qingqing Yin and Li Li are contributed equally to this work

1 Department of Pathogen Biology, Key Laboratory of Prevention.

and Control for Emerging Infectious Diseases of Guangdong Higher

Institutes, Guangdong Provincial Key Laboratory of Tropical Disease

Research, School of Public Health, Southern Medical University,

Guangzhou 510515, Guangdong, China

Full list of author information is available at the end of the article
}

(c) The Author(s) 2019. This article is distributed under the terms of the Creative Commons Attribution 4.0 International License (http://creativecommons.org/licenses/by/4.0/), which permits unrestricted use, distribution, and reproduction in any medium, provided you give appropriate credit to the original author(s) and the source, provide a link to the Creative Commons license, and indicate if changes were made. The Creative Commons Public Domain Dedication waiver (http://creativecommons.org/ publicdomain/zero/1.0/) applies to the data made available in this article, unless otherwise stated. 
for improving MBD risk assessments as well as practical strategies for vector control. For instances of all-day-long frequent female biting during the favorable periods in Guangzhou, effective integrated mosquito control measures must be taken throughout the day and night.

Keywords: Aedes albopictus, Hourly host-seeking behavior, Climatic variables, Mosquito-borne diseases, Field-based modeling, Female biting behavior

\section{Background}

Mosquito-borne diseases (MBD), including dengue, Zika, chikungunya and yellow fever, have been considered an increasing public health challenge worldwide in concert with the rapid spread of Aedes aegypti and Ae. albopictus mosquitoes in recent decades [1,2]. Approximately 390 million annual dengue virus (DENV) infections have been estimated globally [3]. Since the re-emergence of dengue in Foshan, Guangdong, in 1978, dengue outbreaks have been increasing in China, primarily in Guangdong Province $[4,5]$. Guangzhou, the capital city of Guangdong Province has a subtropical monsoon climate, with an average annual temperature of $22-23^{\circ} \mathrm{C}$, and an average rainfall of $1983 \mathrm{~mm}$, which are suitable for MBD transmission in the favorable seasons. For example, there were 38,036 dengue cases reported in Guangzhou in 2014, accounting for $80.8 \%$ of all cases in the largest dengue outbreak in mainland China since 1990 [6]. Aedes albopictus ranks among the top 100 invasive species worldwide and transmits DENV as well as a wide range of arboviruses [7]. The native Asian populations of Ae. albopictus have been inferred to split into three main clusters in equatorial Malaysia and tropical Thailand, subtropical south China and temperate Japan [8]. This mosquito species in Guangzhou belongs to the cluster in subtropical south China [8]. It is a vital vector responsible for dengue outbreaks in Guangzhou [6, $8,9]$ and a potential vector for Zika virus transmission [10]. Both MBD transmission and mosquito populations vary across seasons in local environments and ecological contexts. In Guangzhou, population densities of $A e$. albopictus increase and peak during April and November as estimated using multiple sampling methods [Breteau index (BI), container index (CI), route index (RI), ovitrap index (OI) and adult mosquito density index (ADI)] $[5,9,11-14]$. Meanwhile, the indigenous dengue cases in the region increase and peak from August to November, which lag the active duration of population densities of Ae. albopictus $[5,6]$.

The biting behaviors of female mosquitoes are of crucial importance for MBD transmission. Female biting behavior, population size and seasonal dynamics, longevity, dispersal capacity, and vector competence are the key population parameters for mosquito-based surveillance, which provides a basis for further estimations of vectorial capacity and MBD risk (see [15] for a review). The human biting rate (HBR) is an essential indicator of female biting behaviors and is commonly applied in MBD risk forecasting and assessments of vector interventions [16]. Although an in-depth exploration has been performed to find alternative non-exposure and safe mosquito collection methods, the ADI, which is calculated using human landing collection (HLC), remains the standard for providing consistent and reliable HBRs [17]. The humanbaited double net trap (HDN) has been verified as a safer alternative to HLC for Ae. albopictus monitoring [18, 19]. Thus, the HBRs of wild Ae. albopictus populations acquired via HDNs or HLC can be utilized for evaluating MBDs and vector control strategies as well as risk forecast modeling such as estimations of the basic reproduction number $\left(R_{0}\right)$ of MBDs transmission under the Ross-Macdonald model framework [20, 21].

The climatic variables associated with hourly host-seeking behaviors in wild populations of Ae. albopictus play a key role, especially in establishing a complex forecast model of MBD transmission. Under laboratory conditions, Ae. albopictus is verified as a temperature-sensitive mosquito species, with no biting activity occurring below $11{ }^{\circ} \mathrm{C}$ or above $36^{\circ} \mathrm{C}$ [22]. The survival times of adult $A e$. albopictus at different temperatures observed in the laboratory could be useful in predicting the dynamics and spread of the population [22]. Although the complicated biogeoclimatic context results in microhabitat diversity in the field, the bioecology, spatiotemporal distribution and vectorial capacity of mosquito vectors have been shown to be constrained by factors such as temperature, relative humidity, wind velocity, light intensity, rainfall, land use, vector control measures and human population density, which in turn affect the spread of MBD [23-26]. With global warming, rapid globalization and urbanization in the past 30 years, dengue transmitted by Aedes has been regarded as the fastest spreading arboviral disease [27]. However, the current ecological pattern of hourly host-seeking behaviors of Ae. albopictus, together with its association with climatic variables in the outdoor environments, is still not well demonstrated.

In addition, the combination of incompatible and sterile insect techniques (IIT-SIT) provides a promising approach for MBD control, presenting the near elimination of the wild population of Ae. albopictus in 
test islands in Guangzhou [28]. It is accessible for us to illustrate the temporal pattern of hourly activity in wild female and male Ae. albopictus populations, which makes it possible to select an appropriate time period for releasing the IIT-SIT males.

Modeling is useful for representing conventional bioecological knowledge in statistical or mathematical frameworks and thus it can be used to identify the influential parameters in MBD transmission and provide a quantitative description [21, 29, 30]. Therefore, we undertook a field-based statistical modeling study to explore the ecological characteristics of hourly host-seeking behavior of Ae. albopictus and its associated climatic variables including temperature, relative humidity, illuminance and wind speed in urban outdoor environments in Guangzhou.

\section{Methods}

\section{Field investigations using HDNs}

HDNs (Guangzhou Beikang Biological Vector Monitoring Equipment Company, Guangzhou, China) were selected for the hourly observations in urban outdoor environments in Guangzhou, which included multi-month and multi-site investigations. To explore hourly host-seeking activity patterns of Ae. albopictus, and their relationship with site-specific climatic variables, multi-month investigations were designed to account for all weather conditions and lasted for 13 months excluding rainy and/or strong windy days. Meanwhile, hourly observations were conducted in four similar urban outdoor microhabitats during the favorable period with a high mosquito abundance, named multi-site investigations. On each investigation day, hourly observations were performed for 15 min per hour for $24 \mathrm{~h}$ [31]. Thereby, a total of 912 hourly observations were recorded, including the multimonth investigations with two repetitions per month at 4-10 days apart $(24 \mathrm{~h} \times 2$ repetitions $\times 13$ months $=624)$ from November 2016 to November 2017 and the multisite investigations with three repetitions per site at $4-10$ days apart $(24 \mathrm{~h} \times 3$ repetitions $\times 4$ sites $=288)$ during June-July 2018. Mosquito samples and a total of 912 sets of data on mosquito hourly density and site-specific climatic variables including the hourly ambient temperature, relative humidity, illuminance and wind speed were collected simultaneously. The eligible site selection and the process of hourly observation are described in detail in Additional file 1: Text S1.

In urban areas of Guangzhou, the site $\left(23^{\circ} 11^{\prime} 13^{\prime \prime} \mathrm{N}\right.$, $113^{\circ} 19^{\prime} 38^{\prime \prime}$ E), named the Main Site, was selected for the multi-month and multi-site investigations, while another three sites, named Site A $\left(23^{\circ} 11^{\prime} 21^{\prime \prime} \mathrm{N}\right.$, $\left.113^{\circ} 19^{\prime} 52^{\prime \prime} \mathrm{E}\right)$, Site B $\left(23^{\circ} 9^{\prime} 30^{\prime \prime} \mathrm{N}, 113^{\circ} 21^{\prime} 7^{\prime \prime} \mathrm{E}\right)$ and Site C $\left(23^{\circ} 9^{\prime} 26^{\prime \prime} \mathrm{N}, 113^{\circ} 20^{\prime} 59^{\prime \prime} \mathrm{E}\right)$, were selected for the multisite investigations (Fig. 1). Hourly host-seeking activities of adult mosquitoes in the field observed by HDNs were calculated according to the formula: $D$ (hourly density of adult mosquitoes, mosquito number per person per hour) $=N$ (the total number of adult mosquitoes collected by the 2 collectors in $15 \mathrm{~min}$ )/[the 2 collectors $\times(15 \mathrm{~min} / 60 \mathrm{~min}$ per hour $)]$.

\section{Mosquito species identification}

Mosquitoes sampled by the field investigations were frozen at $-20{ }^{\circ} \mathrm{C}$ for $15 \mathrm{~min}$ and morphological species identification was carried out using the taxonomic key by $\mathrm{Lu}$ et al. [32]. Further species identification of mosquitoes was confirmed by PCR using the mitochondrial cytochrome $c$ oxidase subunit 1 gene $(\operatorname{cox} 1)$ and its sequence analyses (Additional file 2: Text S2).

\section{Statistical modeling for characterization of hourly host-seeking behaviors in wild Ae. albopictus populations}

Kernel regression models were used to assess the temporal patterns of the time-series of hourly female and male Ae. albopictus densities across time points within a day (TPWADs: 0:00, 1:00, ....., 23:00 h). A bootstrap method was used to estimate the $95 \%$ confidence interval of smoothed adult mosquito hourly host-seeking density. Since previous studies reported that a wild population of Ae. albopictus from Guangzhou presented significant seasonal dynamics $[5,9,11-14]$, we separately examined the temporal patterns in two different periods: the favorable period in November 2016 and from April to November 2017, and the unfavorable period from December 2016 to March 2017.

We explored the association between hourly hostseeking activities of female and male Ae. albopictus by examining the association of short-term fluctuations. Considering the consistent temporal patterns of the time-series of female and male mosquito densities across months and across TPWADs could bias their short-term associations, we filtered the time-series of mosquito densities using negative binomial regression models with a categorical variable for the month (Month) and TPWAD as the independent variables. Models with an additional random-effect variablesampling site were used to filter the time-series of adult mosquito densities, which were collected during JuneJuly 2018. Then, we used Spearman's rank correlation coefficients to assess the associations between the filtered time-series of female and male mosquito hourly densities. 


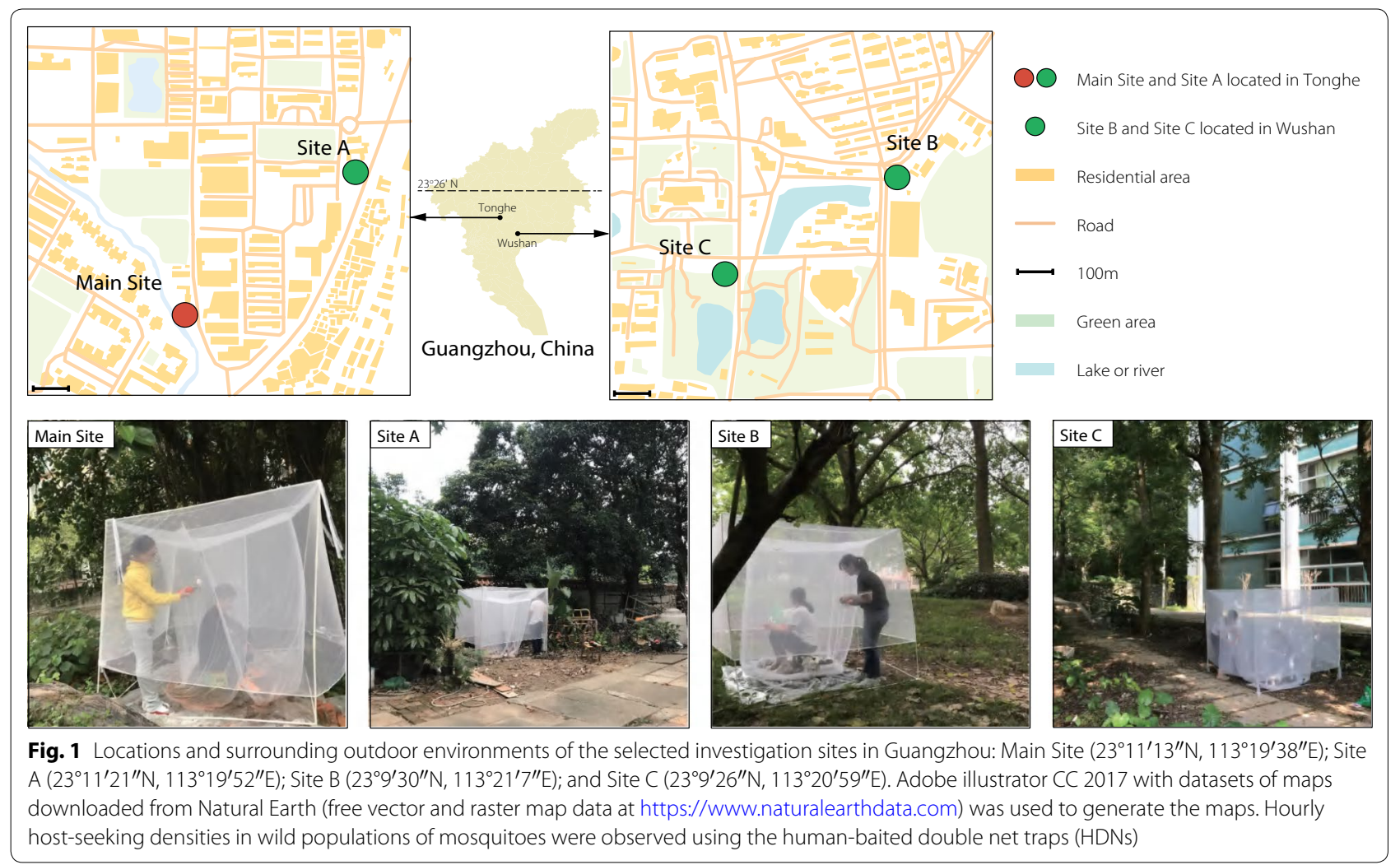

\section{Statistical modeling for assessing the relationship between climatic variables and hourly host-seeking behaviors in wild Ae. albopictus populations}

Given that values of mosquito hourly density followed a negative binomial distribution, we applied negative binomial regression models in the Bayesian framework to assess the associations between hourly hostseeking behaviors of mosquitoes and their associated climatic variables, including hourly densities of female and male Ae. albopictus, with temperature, relative humidity, illuminance and wind speed. Our aim was to understand the role of potential influential factors on mosquito hourly host-seeking activity in the field, yet sample counts are also influenced by population size. Population size varies on a timescale of months because the generation length of Ae. albopictus is approximately 20-30 days [33]. We therefore fitted the models both with and without Month as an explanatory variable to help understand the role of potential influential factors. Month probably influenced mosquito densities as well as climatic variables, and thus was likely to be a confounder of their associations. Since a potential confounder should be always considered in the analysis [34], the models with Month were then used in our main analysis. The variable of TPWAD was also included into the models. The candidate independent variables (it is not necessary to include all of the candidate independent variables in the model) included temperature (Temp), relative humidity $(R H)$, illuminance (Illum), whether the time point was in the daytime or at nighttime $\left(d \_\right.$or_n $)$and wind speed (Wind). The model for the period between November 2016 and November 2017 is as follows:

$$
\begin{aligned}
& Y_{t} \sim \operatorname{NB}\left(r, p_{t}\right) \\
& E\left(Y_{t}\right)=\mu_{t}=\frac{r\left(1-p_{t}\right)}{p_{t}} \quad \operatorname{var}\left(Y_{t}\right)=\mu_{t}+\frac{1}{r} \mu_{t}^{2} \\
& \log \left(\mu_{t}\right)=\alpha+\beta_{\text {TPWAD }} n s\left(\text { TPWAD, df }{ }_{\text {TPWAD }}\right)_{t} \\
& \quad+\beta_{\text {Month }} \text { Month }_{t}+\beta_{\text {Temp }} n s\left(\text { Temp }, d f_{\text {Temp }}\right)_{t} \\
& \quad+\beta_{R H} n s\left(R H, d f=d f_{R H}\right)_{t}+\beta_{D N} d \_o r \_n_{t} \\
& \quad+\beta_{\text {Wind }} n s\left(\text { Wind }, d f=d f_{\text {Wind }}\right)_{t}
\end{aligned}
$$

where $Y_{t}$ and $\mu_{t}$ are the observed and expected mosquito hourly densities at time point $t$, respectively; $r$ and $p_{t}$ are parameters of the negative binomial distribution; $n s$ represents the natural cubic spline; $d f$ stands for degrees of freedom; Month $t_{t}$ indicates the vector of a categorical variable for the month at time point $t$; and $\alpha, \beta_{\text {TPWAD }}, \beta_{\text {Month }}$, $\beta_{\text {Temp }}, \beta_{R H}, \beta_{D N}$ and $\beta_{W i n d}$ are vectors of the regression 
coefficients for the intercept, TPWAD, Month, Temp, RH, $d \_o r \_n$ and Wind, respectively.

Since Temp, $R H$ and $d_{-}$or_ $n$ probably mediate the effects of Illum on mosquito hourly density, we fitted a separate model that excluded Temp, RH and $d_{-}{ }_{0} r_{-} n$ to assess the associations between Illum and mosquito hourly densities for the period between November 2016 and November 2017 as follows:

$$
\begin{aligned}
& Y_{t} \sim N B\left(r, p_{t}\right) \\
& E\left(Y_{t}\right)=\mu_{t}=\frac{r\left(1-p_{t}\right)}{p_{t}} \quad \operatorname{var}\left(Y_{t}\right)=\mu_{t}+\frac{1}{r} \mu_{t}^{2} \\
& \log \left(\mu_{t}\right)=\alpha+\beta_{\text {TPWAD }} n s\left(\text { TPWAD,df } \text { TPWAD }_{t}\right. \\
& \quad+\beta_{\text {Month }} \text { Month }_{t}+\beta_{\text {Illum }} n s\left(\log (\text { Illum }+0.001), d f_{\text {Illum }}\right)_{t} \\
& \quad+\beta_{\text {Wind }} n s\left(\text { Wind }, d f=d f_{\text {Wind }}\right)_{t}
\end{aligned}
$$

where $\log ($ Illum +0.001$)$ represents the log-transformation of (Illum +0.001$)$ and $\beta_{\text {Illum }}$ is the corresponding vector of regression coefficients. The clustering effects of sampling sites were considered when fitting models to the data collected during June-July 2018.

The negative binomial regression models were fitted to the data in the Bayesian framework using Markov Chain Monte Carlo methods. We assigned N $\left(0,10^{2}\right)$ and half-Cauchy $(0,5)[35]$ as the prior distributions for the fixed-effect and random-effect parameters, respectively. We used a 3-chain run for 1000 warm-up and 4000 post-warm-up iterations and kept every fourth iteration, yielding a total of 3000 posterior samples each model. The histograms of the parameter values (excluding those from warm-up iterations) are presented. Trace plots of the values of parameters (excluding those from warm-up iterations) from each chains and potential scale reduction statistic $\hat{R}$ [36] were used to check the convergence of Markov chain Monte Carlo (MCMC) chains.

The $d f s$ for the TPWAD, Temp, RH, Illum and Wind were selected by the minimum value of the WatanabeAkaike information criterion (WAIC) [37] for the models. If the WAIC values for two models with different $d f \mathrm{~s}$ for one variable were not significantly different, then the most parsimonious model was retained. We included the candidate independent variables one by one in the models. A variable was included into the model if the inclusion of the variable led to a reduction in the WAIC compared with that of the models without the variable. The $R^{2}$ for the Bayesian regression models were provided to indicate the proportion of variance in mosquito density explained by the included independent variables [38]. Specifically, we provided the $R^{2}$ value for models with TPWAD, Month, and one candidate variable (i.e. Temp, $R H$, Illum, $d \_o r \_n$ and Wind) and the $R^{2}$ for final models with an intercept, TPWAD, Month and multiple included variables.
The predicted values of mosquito hourly densities changed with the included independent variables. However, the dose-response curves of mosquito hourly densities and one independent variable for different values of covariates should be parallel. To assess the dose-response relationships between mosquito hourly densities and independent variables, we predicted mosquito hourly densities using all observed values of one included independent variable and setting other independent variables (i.e. covariates) to fixed values. We set the values of continuous covariates to be their medians and TPWAD to be 18:00 $\mathrm{h}$ in June. We predicted mosquito hourly densities for the daytime when examining the dose-response curves of mosquito hourly densities and climatic variables (Additional file 3: Table S1). The posterior distributions of parameters were used to estimate the $95 \%$ credible intervals (CrI) for the predicted mosquito hourly densities.

To explore the possible ranges of temperatures at which adult mosquitoes exhibit biting behavior, we estimated the ranges of temperatures that corresponded to the predicted adult mosquito densities which were $\geq 1$ per person per hour for combinations of different values of independent variables. Specifically, we created all possible combinations of the observed values of climatic variables each month because the observed climatic variables varied across months. Then, we repeated the created combinations each TPWAD, thus forming a design matrix, and thereafter predicted densities of adult mosquitoes for the design matrix. The estimated ranges of temperatures that corresponded to $\geq 1$ predicted densities of adult mosquitoes are presented by month.

Sensitivity analyses were conducted to (i) compare results of the correlation between mosquito hourly densities and climatic variables based on the models with and without Month; (ii) examine the dose-response curves of mosquito hourly densities and climatic variables, including Temp, $R H$ and Wind based on the models with Illum instead of $d_{-}$or_n; and (iii) evaluate the associations between mosquito hourly densities and Illum based on the models with variables including Temp and $R H$.

All analyses were conducted using R v.3.4.3 (R Foundation for Statistical Computing, Vienna, Austria).

\section{Results}

A total of 2852 adult mosquitoes were collected during our multi-month investigations from November 2016 to November 2017 (Additional file 4: Table S2), and two species were identified, Ae. albopictus and $C x$. quinquefasciatus. The majority $(71 \%, 2025 / 2852)$ of the collected mosquitoes were Ae. albopictus, while 29\% (827/2852) 
were Cx. quinquefasciatus (Additional file 4: Table S2). Meanwhile, in the multi-site investigations during JuneJuly 2018, we collected 3938 adult mosquitoes. Aedes albopictus, Cx. quinquefasciatus and Armigeres subalbatus (Additional file 5: Table S3) were identified; Ae. albopictus with a total of 3544 also accounted for the largest proportion. Consequently, constituting $82 \%(5569 / 6790)$ of the total collected mosquitoes, Ae. albopictus presented abundant in outdoor urban environments in Guangzhou, while $C x$. quinquefasciatus was another common mosquito species, accounting for $17.6 \%(1193 / 6790)$, which is consistent with previous reports $[9,39,40]$. The total numbers of all collected mosquitoes during daytime and nighttime were, respectively, 2300 and 929 females and 1807 and 533 males of Ae. albopictus, and 160 and 819 females and 80 and 134 males of $C x$. quinquefasciatus. In accordance with previous studies, the hourly host-seeking behavior of $C x$. quinquefasciatus showed a dominant nocturnal pattern (Additional file 4: Table S2, Additional file 5: Table S3). Further statistical modeling of $C x$. quinquefasciatus was limited by its lower number of collected samples using HDNs in this study. Although the predicted hourly densities of female and male Ae. albopictus were still observed to be higher during the daytime than during the nighttime [33] (Fig. 2, Additional file 4: Table S2, Additional file 5: Table S3, Additional file 6: Table S4), our field-based modeling indicated that hourly host-seeking behavior of $A e$. albopictus exhibited a complex pattern as the following demonstration shows in detail.

\section{Ecological characteristics of hourly host-seeking behaviors in Ae. albopictus revealed by the field-based modeling study}

In the present study, hourly host-seeking behaviors of $A e$. albopictus represented a complex pattern with significant variations on an hourly basis (Figs. 3, 4) and generally varied across months and sites.

In the multi-month investigations, hourly densities ranged between 0-78 (mean of 4) and 0-58 (mean of 2) for female and male Ae. albopictus, respectively (Figs. 2a, 3). Consistent with the seasonal variations in population densities of Ae. albopictus, as well as dengue morbidity in Guangzhou reported in previous studies [5, 6, 9, 11-14], hourly host-seeking activities of Ae. albopictus also presented a seasonal variation (Additional file 7: Figure S1), lower during the unfavorable period from December 2016 to March 2017 (Figs. 2a, 3a, b), but higher during the favorable period in November 2016 and April to November 2017, especially from May to July 2017 (Figs. 2a, 3c, d).

During the favorable period with high mosquito abundance from June to July 2018, the multi-site investigations further demonstrated that hourly densities were higher, ranging between $0-138$ (mean of 13) and 0-156 (mean of 11) for female and male Ae. albopictus, respectively (Figs. 2b, 4). Although the four sites were carefully selected based on the same selection criteria, heterogeneous hourly activities of Ae. albopictus were observed between Tonghe (Main Site and Site A, $23^{\circ} 11^{\prime} \mathrm{N}, 113^{\circ} 19^{\prime} \mathrm{E}$ ) and Wushan (Site B $23^{\circ} 9^{\prime} \mathrm{N}, 113^{\circ} 21^{\prime} \mathrm{E}$ and Site $\mathrm{C} 23^{\circ} 9^{\prime} \mathrm{N}, 113^{\circ} 20^{\prime} \mathrm{E}$ ) (Figs. 2b, 4).

Aedes albopictus is commonly known as a predominantly diurnal biting species [41], but we found that host-seeking activities of Ae. albopictus occurred frequently all day long during the favorable period through both the multi-month and multi-site field-based modeling analyses (Figs. 2, 3, 4). In the multi-month investigations, the highest hourly biting densities of females during the day and night were 78 and 58, respectively, occurring in June 2017. Meanwhile, in the multi-site investigations, the highest female hourly biting densities reached 138 and 78 during the daytime and nighttime, respectively.

In the multi-month investigations, the peak period of smoothed hourly densities of female Ae. albopictus occurred between 16:00 and 18:00 h, and a small peak occurred between 6:00 and 8:00 h from April to November (Fig. 3c). During the unfavorable period from December 2016 to March 2017, the peak time was from 14:00 to $16: 00 \mathrm{~h}$ (Fig. 3a). Hourly host-seeking activities over 24 hours were also analyzed in the male mosquito populations (Fig. 3b, d). Double peaks were observed in the smoothed hourly densities of male, with the first peak occurring between 05:00 and 08:00 $\mathrm{h}$ and the second peak occurring between 16:00 and 18:00 $\mathrm{h}$ in the favorable period (Fig. 3d). Meanwhile, in the multi-site investigations, the smoothed hourly density of females peaked between 05:00 and 08:00 $\mathrm{h}$ and between 16:00 and 19:00 h, with variations among the four sites (Figs. 2b, 4a). Double peaks were also observed in the smoothed hourly densities of males, with the first peak occurring between 05:00 and 07:00 $\mathrm{h}$ and the second peak occurring between 16:00 and 18:00 h (Figs. 2b, 4b).

Interestingly, we found a short-term association between the hourly activity of female and male mosquitoes by both the multi-month and multi-site modeling analyses (between November 2016 and November 2017: $r_{s}=0.545, N=624, P<0.001$; June and July 2018: $r_{s}=0$. $470, N=288, P<0.001)$.

\section{Association analyses between hourly host-seeking activity} of adult Ae. albopictus and climatic variables

In the multi-month investigations, the site-specific temperature, relative humidity, illuminance and wind speed 


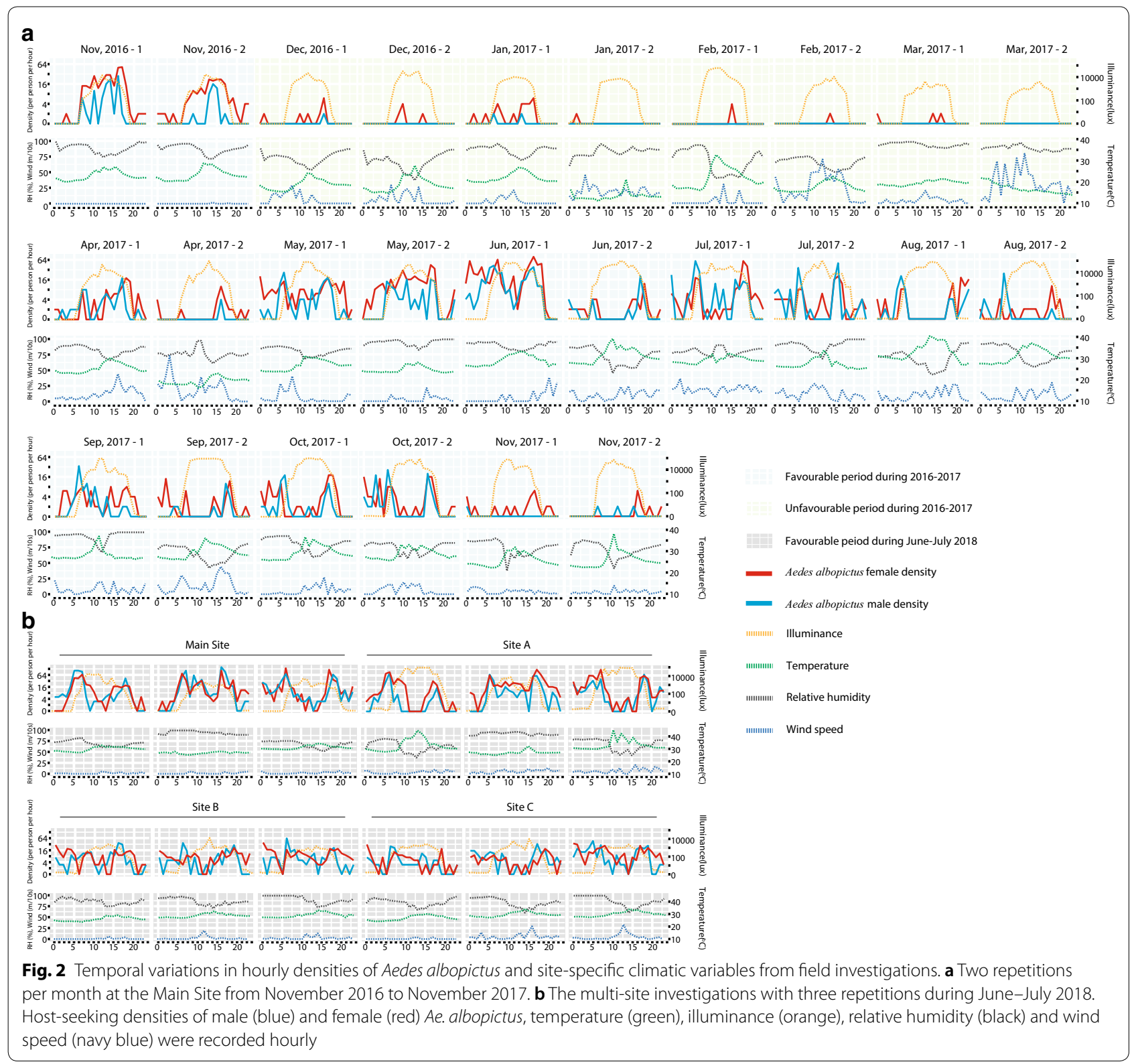

ranged between $11.1-40.4{ }^{\circ} \mathrm{C}, 37-99 \%, 0-116,000$ lux and $0-7.9 \mathrm{~m} / \mathrm{s}$, respectively, with means of $24.7 \pm 5.8^{\circ} \mathrm{C}$, $79.5 \pm 13.5 \%, 10,115.71 \pm 22,288.20 \mathrm{lux}$ and $0.9 \pm 1.2 \mathrm{~m} / \mathrm{s}$, respectively (Fig. 2a). Two final models for females were constructed for the period between November 2016 and November 2017: (i) a model with an intercept, TPWAD, Month, Temp, RH, d_or_n and Wind (Additional file 8: Table S5a); and (ii) a model with an intercept, TPWAD, Month, Illum and Wind (Additional file 8: Table S5b). The independent variables of the two final models for females respectively explained $45.6 \%$ (95\% CrI: $34.7-$ $54.5 \%$ ) and $46.3 \%$ (95\% CrI: $35.0-54.2 \%$ ) of the variance in hourly densities of females. The two final models for males were fitted for the period between November
2016 and November 2017: (i) a model with an intercept, TPWAD, Month, Temp, $R H$ and $d \_o r \_n$ (Additional file 8: Table S5c); and (ii) a model with an intercept, TPWAD, Month, and Illum (Additional file 8: Table S5d). The included independent variables of the two final models for males respectively explained 58.5\% (95\% CrI: $44.2-$ 64.2\%) and 51.5\% (95\% CrI: 35.4-58.5\%) of the variance in hourly densities of males. The point estimate of $R^{2}$ for the model with Illum for females was higher than those for the models with other variables, but the $95 \% \mathrm{CrI}$ of the $R^{2}$ values for models with different variables overlapped (Additional file 9: Table S6).

The temperature, relative humidity, illuminance and wind speed, which were recorded in the multi-site 

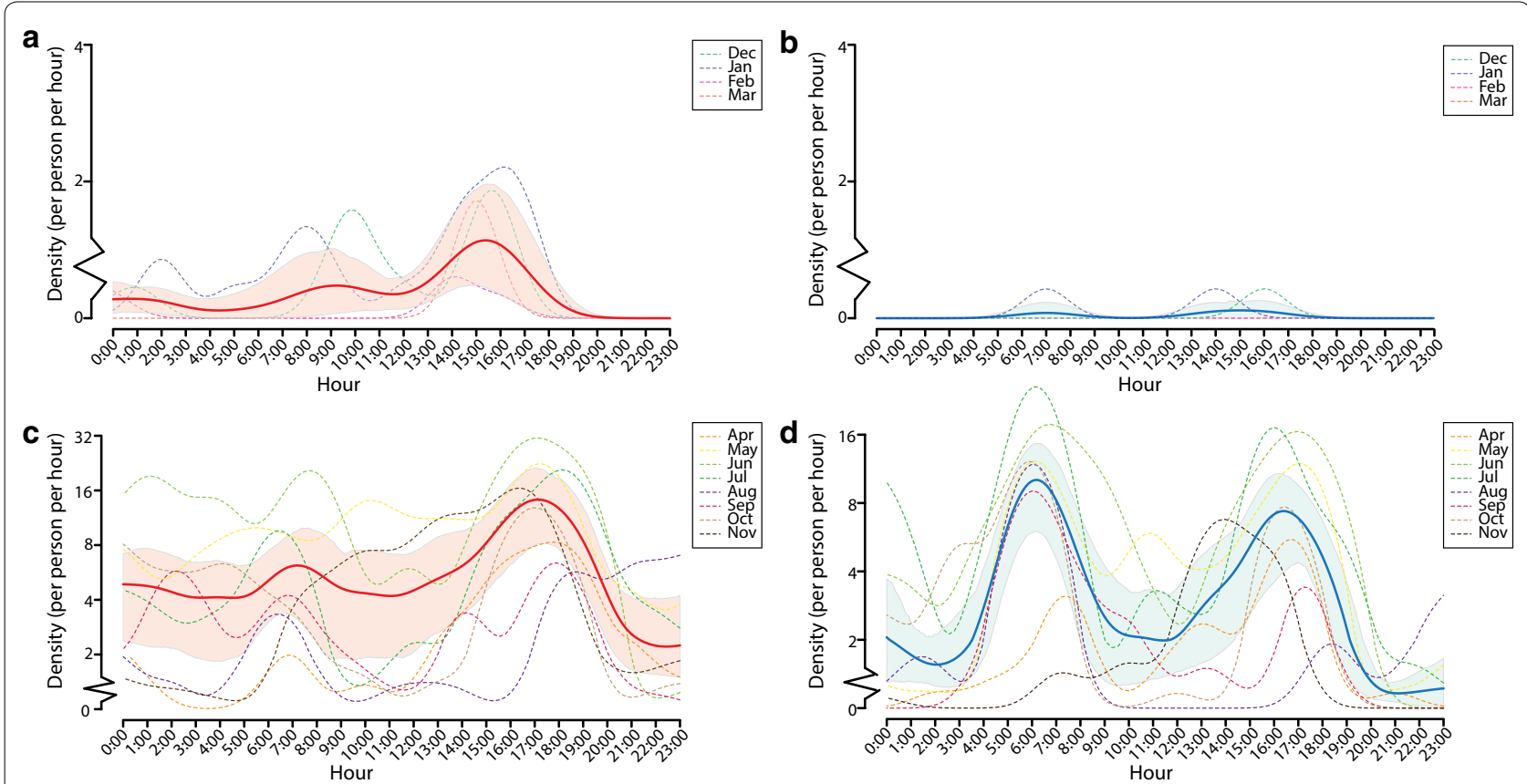

Fig. 3 Temporal patterns in hourly host-seeking activities of Aedes albopictus from multi-month investigations. a-d Hourly dynamics of host-seeking behaviors found in female (a) and male (b) Ae. albopictus from multi-month investigations during the unfavorable period in $2016-$ 2017 and female (c) and male (d) Ae. albopictus from multi-month investigations during the favorable period in 2016-2017. The smoothed hourly densities of female and male Ae. albopictus are displayed in solid red and blue lines, respectively. The shaded areas represent 95\% credible intervals of the smoothed hourly densities
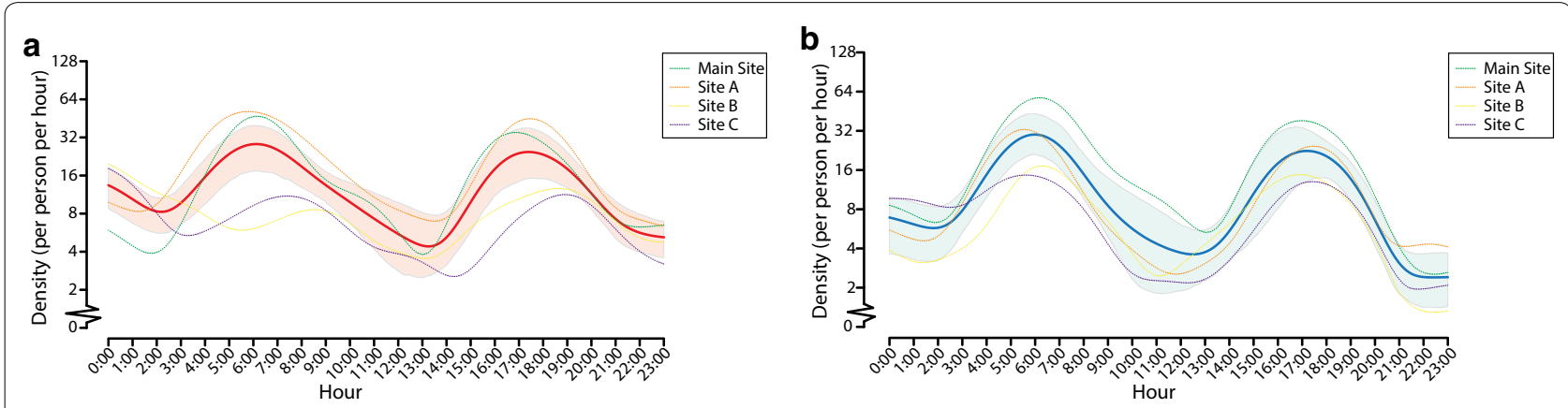

Fig. 4 Temporal patterns in hourly host-seeking activities of Aedes albopictus from multi-site investigations. a, b Hourly dynamics of host-seeking behaviors found in female (a) and male (b) Ae. albopictus from multi-site investigations during June-July 2018. The smoothed hourly densities of female and male Ae. albopictus are displayed in solid red and blue lines, respectively. The shaded areas represent $95 \%$ credible intervals of the smoothed hourly densities

investigations, ranged between $23.6-45.1^{\circ} \mathrm{C}, 37.0-99.0 \%$, 0.0-91,200.0 lux and $0.0-3.3 \mathrm{~m} / \mathrm{s}$, respectively, with means of $29.3 \pm 3.3{ }^{\circ} \mathrm{C}, 80.6 \pm 13.8 \%, 4383.83 \pm 15,583.14$ lux and $0.3 \pm 0.4 \mathrm{~m} / \mathrm{s}$, respectively (Fig. $2 \mathrm{~b}$ ). The mean temperature and illuminance were higher at the Main Site and Site A than at Site B and Site C. Two final models were constructed for females and males during JuneJuly 2018: (i) a model with an intercept TPWAD, Month, Temp and $d \_o r \_n$, (Additional file 10: Table S7a, b); and (ii) a model with an intercept TPWAD, Month and Illum
(Additional file 10: Table S7c, d). The included independent variables of the two final models for females respectively explained $19.2 \%$ (95\% CrI: $1.4-50.8 \%$ ) and 5\% (95\% CrI: $0.3-42.8 \%)$ of the variance in hourly densities of females, and the included independent variables of the two final models for male respectively explained $29.4 \%$ (95\% CrI: $1.9-50.5 \%)$ and 7.7\% (95\% CrI: $0.5-38.3 \%)$ of the variance in hourly densities of males.

In the multi-month investigations, the dose-response curves of the mosquito hourly densities and independent 
variables are presented in Fig. 5. The predicted hourly female and male densities initially increased with temperature, peaking at $26.5^{\circ} \mathrm{C}$ (95\% CrI: $\left.25.3-28.1\right)$ and 28.4 ${ }^{\circ} \mathrm{C}$ (95\% CrI: 27.0-30.4), respectively, and then decreased with temperature (Fig. 5a, e). Similarly, the predicted hourly female and male densities also initially increased with illuminance, peaking at 348.20 lux (95\% CrI: 136.49825.81) and 221.49 lux (95\% CrI: 68.74-555.72), respectively, and then decreased with illuminance (Fig. 5b, f). Meanwhile, the predicted hourly female and male densities increased with increasing relative humidity and generally decreased with increasing wind speed (Fig. 5c, d, g).

Similar associations of adult mosquito hourly density with independent climatic variables were further verified by the multi-site investigations (Fig. 6). During JuneJuly 2018, the predicted hourly female and male densities initially peaked and then decreased with temperature fluctuation (Fig. 6a, c). Meanwhile, the predicted hourly female and male densities also initially increased with illuminance, peaking at 11.93 lux (95\% CrI: 0.35-187.17) and 369.15 lux (95\% CrI: 179.59-602.72), respectively, and then decreased with illuminance (Fig. 6b, d). Notably, relative humidity and wind speed were not included in the models for the multi-site investigations because the inclusion of these variables would not lead to a reduction in the WAIC. Trace plots of the parameter values suggested that the MCMC chains have attained stationarity and mixed, which indicated the convergence of MCMC chains (Additional file 11: Figure S2). $\hat{R}<1.1$ also suggested that the MCMC chains were converged.
The estimated range of temperatures corresponding to predicted hourly densities $\geq 1$ per person per hour for Ae. albopictus found by the multi-month modeling are presented in Additional file 12: Table S8. The minimum and maximum temperatures that corresponded to a predicted female biting density $\geq 1$ per person per hour were 16.4 and $37.1{ }^{\circ} \mathrm{C}$, respectively, whereas those that corresponded to a predicted male density $\geq 1$ per person per hour were 21.7 and $37.1^{\circ} \mathrm{C}$, respectively.

The sensitivity analyses indicated that (i) month was likely to be a confounder of the associations between mosquito densities and climatic variables given the differences observed in their estimated associations using the models with and without Month (Figs. 5, 6 and Additional file 13: Text S3a, b); (ii) the dose-response curves of mosquito hourly densities and climatic variables, including Temp, RH and Wind estimated from the models with Illum but without $d_{-}$or_n $n$ were similar to those in the main analysis (Additional file 13: Text S3c, d); and (iii) Temp and $R H$ could mediate the effects of Illum on mosquito hourly densities given the differences observed between the models with and without Temp and $R H$ (Additional file 13: Text S3e).

\section{Discussion}

In the present field-based modeling, the ecological characteristics of hourly host-seeking behaviors of Ae. albopictus and the associated site-specific climatic variables including temperature, illuminance, relative humidity and wind speed were systematically clarified. The results

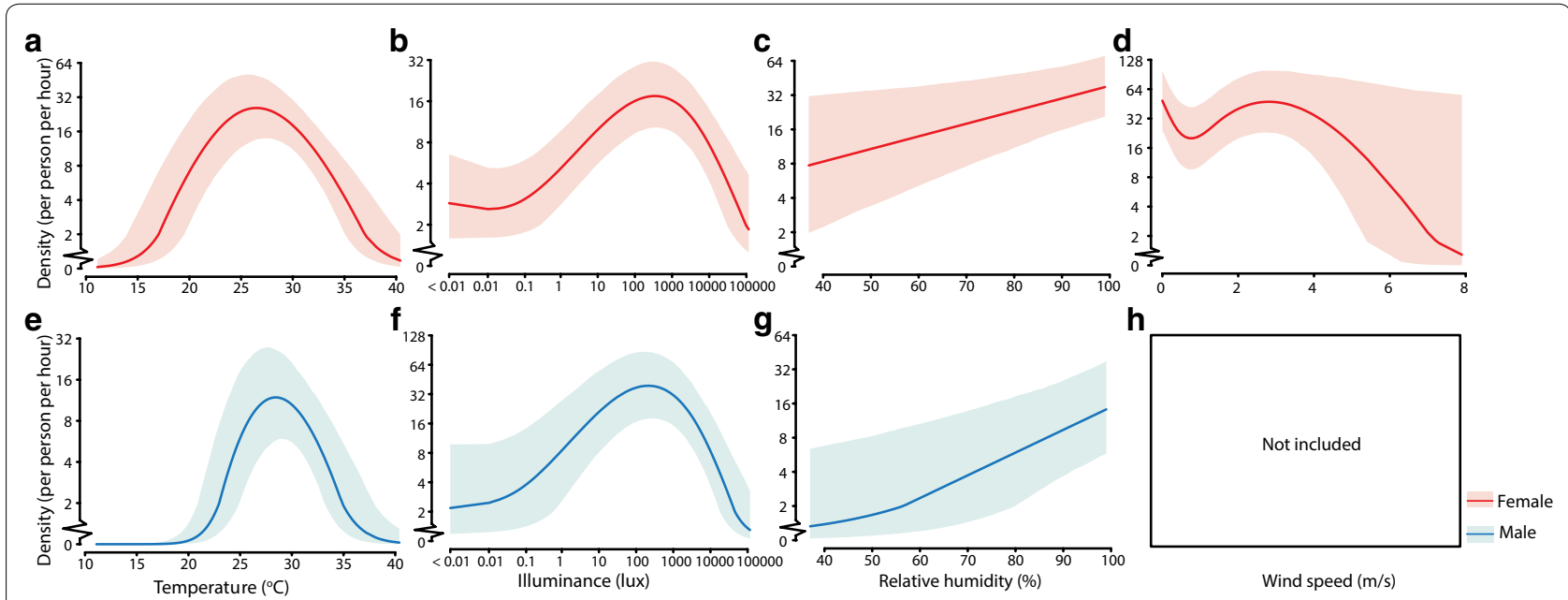

Fig. 5 Dose-response relationships between hourly host-seeking activities of Aedes albopictus and climatic variables from multi-month investigations. The solid lines represent the predicted values of Ae. albopictus hourly densities by temperature, illuminance, relative humidity and wind speed, assuming that the other continuous covariates were equal to their medians and that the time point was at 18:00 $\mathrm{h}$ in June. The shaded areas represent the $95 \%$ credible intervals of the predicted hourly densities. Dose-response relationships between hourly host-seeking activities of female (a-d), male (e-h) Ae. albopictus and temperature, illuminance, relative humidity and wind speed in multi-month investigations from November 2016 to November 2017 
a
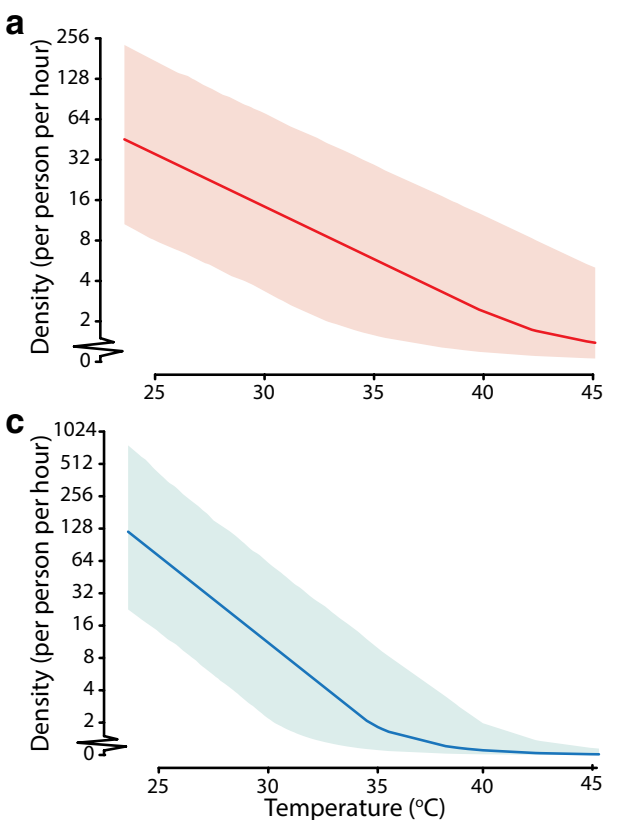

b

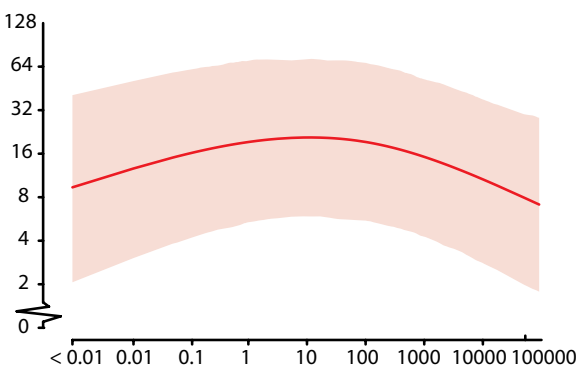

d

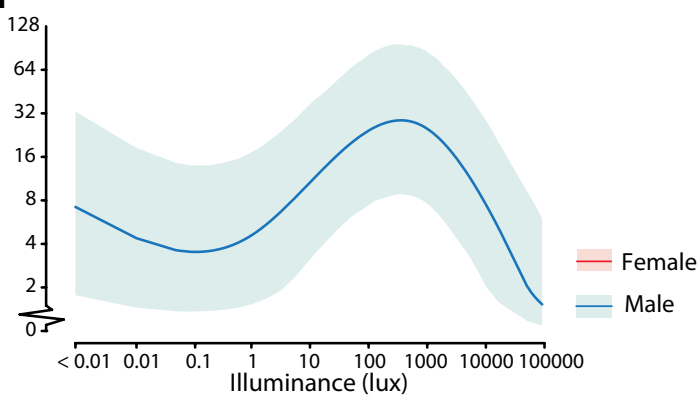

Fig. 6 Dose-response relationships between hourly host-seeking activities of Aedes albopictus and temperature, illuminance from multi-site investigations. The solid lines represent the predicted values of Ae. albopictus hourly densities by temperature and illuminance, assuming that the other continuous covariates were equal to their medians and that the time point was at 18:00 $\mathrm{h}$ in June. Dose-response relationships between hourly host-seeking activities of female $(\mathbf{a}, \mathbf{b})$, male $(\mathbf{c}, \mathbf{d})$ Ae. albopictus and temperature and illuminance in multi-site investigations during JuneJuly 2018

demonstrated that hourly host-seeking behaviors of Ae. albopictus present a complex pattern with an hourly variation and are significantly influenced by climatic variables.

The seasonal fluctuations in the population densities of Ae. albopictus and dengue morbidity have been recorded in Guangzhou [5, 6, 9, 11-14]. The divergence of hostseeking behavior in Ae. albopictus across site-specific microhabitats was also described in earlier observations $[33,42]$. Similar to these previous studies, the heterogeneity and complexity of host-seeking behavior of $A e$. albopictus were confirmed across sites (Figs. 2b, 4) and months (Figs. 2a, 3). For instance, divergence across places was found from Tonghe to Wushan in our multisite investigations. The hourly variation in host-seeking behavior of Ae. albopictus was likely attributable to all variances of the influential factors including time points, microhabitats and the inherent bioecological characteristics of Ae. albopictus and was crucially constrained by climate variances.

Hourly variation in host-seeking behavior of Ae. albopictus exhibited bimodality and female biting occurred frequently all day long during the favorable period

As is known, Ae. albopictus usually bites during daytime [41]. However, during the favorable period both in the multi-month and multi-site investigations in urban outdoor environments, we found that hourly host-seeking activities of adult Ae. albopictus occurred frequently all day long, and that females in particular presented aggressive biting behavior throughout the day and night. Although the hourly densities were higher during the day, female biting also occurred frequently at night. During June-July 2018, the female hourly biting density reached up to 78 at night. Aedes albopictus is strongly anthropophilic and has a higher blood-feeding rate in urban areas, where the human population density is greater [43]. The environmental changes caused by urbanization have had a considerable impact on the ecology of Ae. albopictus [44]. The more frequent nighttime biting of female Ae. albopictus in urban outdoor environments in Guangzhou during the favorable periods may be attributed to complex factors, including increasing human population density, more larval breeding habitats, bright city lights and changing climatic conditions due to global warming. The all-day-long biting activity of females may pose an increasing risk of dengue transmission. Therefore, effective measures must be taken to prevent the biting activity of Ae. albopictus throughout both the day and night.

In addition, previous observations also discovered a bimodal pattern of female Ae. albopictus biting: (i) one peak at dawn and one peak occurring in the afternoon, 
with lower nonzero activity levels between these two peaks in Macao, China [45]; (ii) the highest host-seeking activity peak occurring approximately two hours before sunset and a smaller but important activity peak occurring at 8:30 h on La Réunion Island in the Indian Ocean [46]. Documented in a monograph on dengue fever and its vectors in China [33], field investigations focusing on the biting behavior of Ae. albopictus had been launched in Fuzhou, Fujian Province (Wang, 1952), Shanghai (Liu, 1958), and six sites in Yixing, Jiangsu Province; Nanyang and Boai, Henan Province; and Nanning, Guangxi Province (the National Cooperation Group, China, 1979). Most of the hourly observations using HDNs were performed in outdoor environments, especially in bamboo forest microhabitats with abundant mosquitoes during 04:00-20:00 h or all-day-long. A descriptive analysis of these data indicated that patterns of biting behaviors in the wild populations of Ae. albopictus were similar in different areas in China, indicating this species is a dominant diurnal biter with two biting peaks, the first at one to two hours around the local sunrise and the other at two to three hours before the local sunset, with the latter peak being higher.

In agreement with these previous studies, our fieldbased modeling provided an in-depth quantitative description of the bimodality in hourly host-seeking behavior of Ae. albopictus. During the favorable period, the highest female biting activity occurred from 16:00 to 18:00 $\mathrm{h}$ based on the modeling estimates from the multimonth investigations. Meanwhile, the multi-site investigations estimated that female biting primarily peaked from 16:00 to 19:00 h. The secondary peaks of female biting occurred from 06:00 to 08:00 $\mathrm{h}$ and 05:00 to 08:00 $\mathrm{h}$, estimated by the multi-month and multi-site fieldbased modeling, respectively. Although it varies slightly depending on different times and locations, the bimodality of female biting behavior is similar to that described previously $[45,46]$. Thus, the bimodality of female biting could be recognized as appearing within two to three hours around both dawn and dusk, i.e. 05:00-08:00 $\mathrm{h}$ and 16:00-19:00 h, in urban outdoor environments in Guangzhou during the favorable period. Therefore, we also call for greater awareness of the high risk of female biting, as well as dengue transmission during the peak times, among governments, health authorities and the general public.

\section{Hourly host-seeking behaviors of Ae. albopictus are significantly influenced by climatic variables}

In our study, field-based negative binomial regression models in the Bayesian framework were employed to identify the associations between hourly host-seeking behaviors of Ae. albopictus and climatic variables. Since month could be a potential confounder of their associations, Month was then included in the main models. We focused on the effects of climatic variables on mosquito activity instead of its population size. The variable patterns of host-seeking behaviors of Ae. albopictus in urban outdoor environments in Guangzhou were found to be significantly influenced by climatic variables.

Previous studies reported that climatic variables, including temperature, rainfall, etc., influenced the spatiotemporal distributions and dynamics of vector populations as well as the transmission of vector-borne diseases [23-25, 33, 47, 48]. Among these factors, temperature plays a crucial role in the establishment of a population, mosquito dynamics and dengue transmission mediated by $\mathrm{Ae}$. albopictus [49-53]. Shen et al. [54] reported that a minimum monthly temperature threshold of $18.25{ }^{\circ} \mathrm{C}$ might have been associated with the dengue incidence from June to November during 2006-2014 in Guangzhou. Xiao et al. [55] estimated in a laboratory study that no transmission might occur below $18{ }^{\circ} \mathrm{C}$ based on their findings from $A e$. albopictus infected orally with a DENV-2 suspension and incubated within a temperature range of $18-36^{\circ} \mathrm{C}$. A nonlinear relationship was also found between temperature and dengue incidence [56]. A systematic review and metaanalysis indicated that the minimum temperature (18.1$\left.24.2{ }^{\circ} \mathrm{C}\right)$ and maximum temperature $\left(28.0-34.5^{\circ} \mathrm{C}\right)$ were associated with increased dengue transmission in tropical or subtropical areas [57]. Duan et al. [22] observed that no biting activity occurred below $11{ }^{\circ} \mathrm{C}$ or above $36^{\circ} \mathrm{C}$ under laboratory conditions in Ae. albopictus collected from habitats in Jiangsu Province, China. In comparison with the temperature thresholds for dengue transmission or female biting of Ae. albopictus described in the previous studies, our field-based modeling further demonstrated that the predicted temperature range suitable for female Ae. albopictus biting in urban outdoor environments in Guangzhou was 16.4 to $37.1{ }^{\circ} \mathrm{C}$, with an estimated peak at temperatures of $26.5{ }^{\circ} \mathrm{C}$ (95\% CrI: $\left.25.3-28.1\right)$. The differences in the temperature threshold estimates can be attributed to the different methodologies as well as the complex interactions of environmental factors in outdoor microhabitats.

A previous study reported that the threshold of light intensity for the activation of nocturnal host-seeking activity was greater than 10 lux in Ae. albopictus [25]. Interestingly, our field-based modeling found that female Ae. albopictus biting peaked under a lower illumination of 11.93 lux (95\% CrI: 0.35-187.17) and 348.20 lux (95\% CrI: $136.49-825.81)$ as estimated by our multi-site and multi-month studies, respectively, and both estimates were close to the light densities from dawn and dusk, which might explain the female biting bimodality that appeared within respective two to three hours around dawn and dusk. 


\section{Short-term association of hourly host-seeking activities between female and male Ae. albopictus}

In our study, more female than male mosquitoes were collected, which is consistent with previous observations using HDNs in Madagascar [58]. Double peaks were also observed in the smoothed values for male density by the kernel regression model, with the first peak occurring between 5:00 and 7:00 or 8:00 h, and the second peak occurring between 16:00 and 18:00 h during the favorable periods in 2016 to 2018 . Hourly hostseeking activity patterns of female and male mosquitoes may be related to their mating behavior. Several studies have shown that the mating patterns of Ae. albopictus were bimodal and diurnal in Macao, China, Samui Island, Thailand and La Reunión Island [45, 46, 59, 60], and the peaks were recorded during 6:00-8:00 $\mathrm{h}$ and 16:00-17:00 $\mathrm{h}$ in Macao [45]. Previous studies have reported that $A e$. aegypti and Ae. diantaeus males were waiting for bloodsucking females in the vicinity of the hosts [61, 62]. We found a short-term association between female and male Ae. albopictus hourly densities, which suggests that the mating behavior of Ae. albopictus in urban outdoor environments in Guangzhou may be associated with female biting. These findings should facilitate improvements to the release strategies of IIT-SIT Ae. albopictus males for vectors and MBDs control [28].

\section{Limitations}

In this field-based modeling study, multi-month investigations included two repetitions each month and multisite investigations for three repetitions each site. We performed 24 hourly observations each investigation day using HDNs, with a total of 912 hourly observations performed and 912 site-specific datasets collected. Although the present field-based modeling study is informational, a further investigation launched in more collection sites for more years may further strengthen the study. In another aspect, we did not estimate the relative importance of each climatic variable even though we researched in the associations between mosquito hourly densities and various climatic variables using negative binomial regression models in the Bayesian framework. Further exploration is needed.

\section{Conclusions}

The present field-based modeling indicated that hostseeking behaviors of Ae. albopictus exhibit a complex pattern with hourly variation. Hourly mosquito densities of Ae. albobictus had a non-linear relationship with temperature and illuminance. At the same time, the densities increased with relative humidity while decreasing with wind speed. During the favorable period in urban outdoor environments in Guangzhou, female Ae. albopictus were shown to bite frequently all day long, with bimodality occurring within two to three hours of both dawn and dusk. These findings lay a foundation for improving MBD risk assessments as well as practical strategies for vector control.

\section{Supplementary information}

Supplementary information accompanies this paper at https://doi. org/10.1186/s13071-019-3715-1.

Additional file 1: Text $\mathbf{S 1}$. The eligible site selection and the process of hourly observation.

Additional file 2: Text S2. Species identification of the collected adult mosquitoes

Additional file 3: Table S1. The fixed values of other variables.

Additional file 4: Table S2. Adult mosquitoes collected by multi-month investigations from November 2016 to November 2017.

Additional file 5: Table S3. Adult mosquitoes collected by multi-site investigations during June-July 2018.

Additional file 6: Table S4. The predicted values for the multi-month and multi-site mosquito densities of Ae. albopictus during both the day and night.

Additional file 7: Figure S1. Seasonal variations of hourly host-seeking activities found in Ae. albopictus during 2016-2017. a, b Seasonal variations of hourly host-seeking activities found in female (a) and male (b) Ae. albopictus. The smoothed hourly densities of female and male Ae. albopictus are displayed in solid red and blue lines, respectively. The shaded areas represent $95 \%$ credible intervals of the smoothed hourly densities.

Additional file 8: Table S5. Values of the Watanabe-Akaike information criterion (WAIC) for models used for the assessment of potential influential factors of Ae. albopictus for the period from November 2016 to November 2017.

Additional file 9: Table S6. $R^{2}$ values for the models with time points within a day and month and different variables.

Additional file 10: Table S7. Values of the Watanabe-Akaike information criterion (WAIC) for models used for the assessment of potential influential factors of Ae. albopictus for the period June-July 2018.

Additional file 11: Figure S2. The histograms and trace plots of the parameter values.

Additional file 12: Table S8. Estimated thresholds of temperatures that corresponded to the predicted Ae. albopictus densities which were $\geq 1$ per person per hour from multi-month investigations from November 2016 to November 2017

Additional file 13: Text S3. Sensitivity analyses.

\section{Abbreviations}

MBD: mosquito-borne disease; DENV: dengue virus; HBR: human biting rate; Bl: Breteau index; Cl: container index; Rl: route index; Ol: ovitrap index; cox1: cytochrome c oxidase subunit 1 gene; ADI: adult mosquito density index; HLC: human landing catch; HDN: human-baited double net trap; IIT-SIT: the combination of incompatible and sterile insect techniques; TPWAD: time points within a day; MCMC: Markov chain Monte Carlo; WAIC: Watanabe-Akaike information criterion; Crl: credible interval.

\section{Acknowledgments}

We would like to acknowledge Yifan Li, Ke Cao, Wanyu Li and Ruili Xie from Southern Medical University, Guangzhou, China, for helping field 
investigations and the University Postgraduate Fellowships (UPF) Scheme of the University of Hong Kong.

\section{Authors' contributions}

Conceived and designed the experiments: $X H Z, C M W$ and $X G C$. Performed the experiments: QQY, YJW, YJL, SW, YCP, QW, XG, TX, TH, DX, WYL, ZYS, SYZ, YD and $Y X$. Analyzed the data: $L L, X H Z, Q Q Y, X G, B Y S, Y J W, S X$ and XNZ. Wrote the manuscript: QQY, XHZ, LL, RKW and DMK. All authors read and approved the final manuscript.

\section{Funding}

This study was supported by the National Key R\&D Programme of China (2016YFC1200500), the Guangzhou Synergy Innovation Key Programme for Health (201803040006, 201508020263), the Science and Technology Planning Project of Guangdong Province of China (2016A020251001), the Guangzhou International Science and Technology Cooperation Programme (2012J5100026), National Training Programme of Innovation and Entrepreneurship for Undergraduates (201612121026) and the Guangdong Province Training Programme of Innovation and Entrepreneurship for Undergraduates (201712121114)

\section{Availability of data and materials}

Data supporting the conclusions of this article are included within the article and its additional files. The datasets used and/or analyzed during the present study are available from the corresponding author upon reasonable request.

\section{Ethics approval and consent to participate}

Participation in this study was fully voluntary. The volunteers were recruited after agreeing to participate and signing a written informed consent form. Although no direct exposure to mosquito female-biting occurs with the use of HDNs, all collectors were trained and provided free medical care if they showed any symptoms suspected to be caused by MBDs. The main collectors included the authors. The experiments using HDNs in this study were approved by the Ethics Commission of Southern Medical University (20160011). No protected species were involved in this study.

\section{Consent for publication}

Not applicable.

\section{Competing interests}

The authors declare that they have no competing interests.

\section{Author details}

1 Department of Pathogen Biology, Key Laboratory of Prevention and Control for Emerging Infectious Diseases of Guangdong Higher Institutes, Guangdong Provincial Key Laboratory of Tropical Disease Research, School of Public Health, Southern Medical University, Guangzhou 510515, Guangdong, China. ${ }^{2}$ WHO Collaborating Centre for Infectious Disease Epidemiology and Control, School of Public Health, Li Ka Shing Faculty of Medicine, The University of Hong Kong, Hong Kong, Hong Kong Special Administrative Region, China. ${ }^{3}$ The School of Foreign Studies, Southern Medical University, Guangzhou 510515, Guangdong, China. ${ }^{4}$ HKBU-NIPD Joint Research Laboratory for Intelligent Disease Surveillance and Control, School of Cyberspace, Hangzhou Dianzi University, Hangzhou 310018, Zhejiang, China. ${ }^{5}$ National Institute of Parasitic Diseases, Chinese Center for Diseases Control and Prevention, WHO Collaborating Center for Tropical Diseases, National Center for International Research on Tropical Diseases, Ministry of Science and Technology, Key Laboratory of Parasite and Vector Biology, Ministry of Health, Shanghai 200025, China.

\section{Received: 27 April 2019 Accepted: 12 September 2019}

Published online: 14 October 2019

\section{References}

1. Kraemer MUG, Reiner RC, Brady OJ, Messina JP, Gilbert M, Pigott DM, et al. Past and future spread of the arbovirus vectors Aedes aegypti and Aedes albopictus. Nat Microbiol. 2019;4:854-63.

2. Guzman MG, Gubler DJ, Izquierdo A, Martinez E, Halstead SB. Dengue infection. Nat Rev Dis Primers. 2016;2:16055.
3. Bhatt $S$, Gething PW, Brady OJ, Messina JP, Farlow AW, Moyes CL, et al. The global distribution and burden of dengue. Nature. 2013;496:504-7.

4. Chen R, Han GZ. Dengue in China: comprehensive phylogenetic evaluation reveals evidence of endemicity and complex genetic diversity. Am J Trop Med Hyg. 2016;94:198-202.

5. Luo L, Liang HY, Hu YS, Liu WJ, Wang YL, Jing QL, et al. Epidemiological, virological, and entomological characteristics of dengue from 1978 to 2009 in Guangzhou. China. J Vector Ecol. 2012;37:230-40.

6. Cheng Q, Jing Q, Spear RC, Marshall JM, Yang Z, Gong P. The interplay of climate, intervention and imported cases as determinants of the 2014 dengue outbreak in Guangzhou. PLoS Negl Trop Dis. 2017;11:e5701.

7. Bonizzoni M, Gasperi G, Chen X, James AA. The invasive mosquito species Aedes albopictus: current knowledge and future perspectives. Trends Parasitol. 2013;29:460-8.

8. Sherpa S, Blum MGB, Capblancq T, Cumer T, Rioux D, Després L. Unravelling the invasion history of the Asian tiger mosquito in Europe. Mol Ecol. 2019;28:2360-77.

9. Li YJ, Su XH, Zhou GF, Zhang H, Puthiyakunnon S, Shuai SF, et al. Comparative evaluation of the efficiency of the BG-Sentinel trap, CDC light trap and mosquito-oviposition trap for the surveillance of vector mosquitoes. Parasit Vectors. 2016;9:446.

10. Liu ZZ, Zhou TF, Lai ZT, Zhang ZH, Jia ZR, Zhou GF, et al. Competence of Aedes aegypti, Ae. albopictus, and Culex quinquefasciatus mosquitoes as Zika virus vectors, China. Emerg Infect Dis. 2017;23:1085-91.

11. Li MQ, Liu YQ, Huang YH, Chen HF, Yao YX, Liu B, et al. Seasonal variation in population density, dengue virus carriage, and dichlorvos resistance of Aedes albopictus in urban and rural areas of Guangzhou city, China. Chin J Vector Biol Control. 2013;24:108-11.

12. Oidtman RJ, Lai S, Huang Z, Yang J, Siraj AS, Reiner RJ, et al. Inter-annual variation in seasonal dengue epidemics driven by multiple interacting factors in Guangzhou, China. Nat Commun. 2019;10:1148.

13. Pan YY, Wu HX, Guo J, Liu QY. Population density prediction of Aedes albopictus in Guangzhou based on autoregressive integrated moving average model. Chin J Vector Biol Control. 2018;29:545-9.

14. Xia D, Guo X, Hu T, Li L, Teng PY, Yin QQ, et al. Photoperiodic diapause in a subtropical population of Aedes albopictus in Guangzhou, China: optimized field-laboratory-based study and statistical models for comprehensive characterization. Infect Dis Poverty. 2018;7:89.

15. Petrić D, Bellini R, Scholte E, Rakotoarivony LM, Schaffner F. Monitoring population and environmental parameters of invasive mosquito species in Europe. Parasit Vectors. 2014;7:187.

16. Brady OJ, Godfray HC, Tatem AJ, Gething PW, Cohen JM, McKenzie FE, et al. Vectorial capacity and vector control: reconsidering sensitivity to parameters for malaria elimination. Trans R Soc Trop Med Hyg. 2016;110:107-17.

17. Service M. A critical review of procedures for sampling populations of adult mosquitos. Bull Entomol Res. 1977;67:343-425.

18. Tangena JA, Thammavong P, Hiscox A, Lindsay SW, Brey PT. The human-baited double net trap: an alternative to human landing catches for collecting outdoor biting mosquitoes in Lao PDR. PLoS One. 2015;10:e138735

19. Gao Q, Wang F, Lv X, Cao H, Zhou JJ, Su F, et al. Comparison of the human-baited double net trap with the human landing catch for Aedes albopictus monitoring in Shanghai, China. Parasit Vectors. 2018;11:483.

20. van den Driessche P, Watmough J. Reproduction numbers and subthreshold endemic equilibria for compartmental models of disease transmission. Math Biosci. 2002;180:29-48.

21. Smith DL, Battle KE, Hay SI, Barker CM, Scott TW, McKenzie FE. Ross, macdonald, and a theory for the dynamics and control of mosquitotransmitted pathogens. PLoS Pathog. 2012;8:e1002588.

22. Duan HL, Yang J, Wang YG, Liu RR, Shi CN, Lu L, et al. The survival times of adult Aedes albopictus under different temperatures. Chin J Vector Biol Control. 2017;28:205-8.

23. Paaijmans KP, Read AF, Thomas MB. Understanding the link between malaria risk and climate. Proc Natl Acad Sci USA. 2009;106:13844-9.

24. Naish S, Dale P, Mackenzie JS, McBride J, Mengersen K, Tong S. Climate change and dengue: a critical and systematic review of quantitative modelling approaches. BMC Infect Dis. 2014;14:167.

25. Kawada H, Takemura SY, Arikawa K, Takagi M. Comparative study on nocturnal behavior of Aedes aegypti and Aedes albopictus. J Med Entomol. 2005;42:312-8. 
26. Liu-Helmersson J, Stenlund H, Wilder-Smith A, Rocklov J. Vectorial capacity of Aedes aegypti: effects of temperature and implications for global dengue epidemic potential. PLoS ONE. 2014;9:e89783.

27. World Health Organization. Dengue: Guidelines for diagnosis, treatment, prevention and control. New ed. Geneva: World Health Organization; 2009

28. Zheng XY, Zhang DJ, Li YJ, Yang C, Wu Y, Liang X, et al. Incompatible and sterile insect techniques combined eliminate mosquitoes. Nature. 2019:572:56-61.

29. Brady OJ, Johansson MA, Guerra CA, Bhatt S, Golding N, Pigott DM, et al. Modelling adult Aedes aegypti and Aedes albopictus survival at different temperatures in laboratory and field settings. Parasit Vectors. 2013;6:351.

30. Focks DA, Daniels E, Haile DG, Keesling JE. A simulation model of the epidemiology of urban dengue fever: literature analysis, model development, preliminary validation, and samples of simulation results. Am J Trop Med Hyg. 1995;53:489-506.

31. Silver JB. Mosquito ecology: field sampling methods. 3rd ed. Dordrecht, Netherlands: Springer; 2008

32. Lu BL, Li BS, Ji SH, Chen HB, Meng QH, Su L, et al. Fauna Sinica, Insecta vol. 8, Diptera: Culicidae 1. Beijing, China: Science Press; 1997.

33. Lu BL, Xu RM, Zhang HC, Fu TR, Chen HB, An JY, et al. Ecological habits of Aedes albopictus. In: Lu BL, editor. The transmission vector and prevention of dengue in China. Guiyang: Guizhou People’s Publishing House; 1990. p. 70-106.

34. HealthKnowledge. Confounding in epidemiological studies. 2017. https ://www.healthknowledge.org.uk/node/803. Accessed 6 Jun 2019.

35. Gelman A. Prior distributions for variance parameters in hierarchical models (comment on article by Browne and Draper). Bayesian Anal. 2006;1:515-34.

36. Gelman A, Carlin JB, Stern HS, Dunson DB, Vehtari A, Rubin DB. Bayesian data analysis. 3rd ed. London: Chapman \& Hall/CRC; 2013.

37. Watanabe S. Asymptotic equivalence of Bayes cross-validation and widely applicable information criterion in singular learning theory. J Mach Learn Res. 2010;11:3571-94.

38. Gelmany A, Goodrichz B, Gabryz J, Alix I. R-squared for Bayesian regression models. 2017. http://www.stat.columbia.edu/ gelman/research/ unpublished/bayes_R2.pdf. Accessed 15 Dec 2018.

39. Zou HB, Song W, Lin HB, Li JL, Wang YF, Li ZQ, et al. Background investigation on mosquitoes at Guangzhou ports along the Zhujiang river. Chin J Front Health Quar. 2014;37:331-5.

40. Liu YS, Liang HY, Huang F, Li GH, Zhai JS, Zhang WD, et al. Investigation on mosquitoes at Luogang ports in Guangzhou from 2008 to 2009. Chin J Front Health Quar. 2010;33:4.

41. Hawley WA. The biology of Aedes albopictus. J Am Mosa Control Assoc Suppl. 1988;1:1-39.

42. Casas MM, Orozco BA, Munoz RM, Ulloa GA, Bond JG, Valle MJ, et al. A new tent trap for monitoring the daily activity of Aedes aegypti and Aedes albopictus. J Vector Ecol. 2013;38:277-88.

43. Valerio L, Marini F, Bongiorno G, Facchinelli L, Pombi M, Caputo B, et al. Host-feeding patterns of Aedes albopictus (Diptera: Culicidae) in urban and rural contexts within Rome province, Italy. Vector Borne Zoonotic Dis. 2010;10:291-4.

44. Li YJ, Kamara F, Zhou GF, Puthiyakunnon S, Li CY, Liu YX, et al. Urbanization increases Aedes albopictus larval habitats and accelerates mosquito development and survivorship. PLoS Negl Trop Dis. 2014;8:e3301.

45. Almeida AP, Baptista SS, Sousa CA, Novo MT, Ramos HC, Panella NA, et al. Bioecology and vectorial capacity of Aedes albopictus (Diptera: Culicidae) in Macao, China, in relation to dengue virus transmission. J Med Entomol. 2005:42:419-28.

46. Delatte H, Desvars A, Bouetard A, Bord S, Gimonneau G, Vourc'H G, et al. Blood-feeding behavior of Aedes albopictus, a vector of chikungunya on La Reunion. Vector Borne Zoonotic Dis. 2010;10:249-58.

47. Fredeen FJ, Mason PG. Meteorological factors influencing host-seeking activity of female Simulium luggeri (Diptera: Simuliidae). J Med Entomol. $1991 ; 28: 831-40$
48. Zarroug IM, Hashim K, Elaagip AH, Samy AM, Frah EA, EIMubarak WA, et al. Seasonal variation in biting rates of Simulium damnosum sensu lato, vector of Onchocerca volvulus, in two Sudanese Foci. PLOS ONE. 2016;11:e150309.

49. Ebi KL, Nealon J. Dengue in a changing climate. Environ Res. 2016;151:115-23.

50. Sang SW, Gu SH, Bi P, Yang WZ, Yang ZC, Xu L, et al. Predicting unprecedented dengue outbreak using imported cases and climatic factors in Guangzhou, 2014. PLoS Negl Trop Dis. 2015:9:e3808.

51. Dhimal M, Gautam I, Joshi HD, O'Hara RB, Ahrens B, Kuch U. Risk factors for the presence of chikungunya and dengue vectors (Aedes aegypti and Aedes albopictus), their altitudinal distribution and climatic determinants of their abundance in central Nepal. PLoS Negl Trop Dis. 2015;9:e3545.

52. Yang HM, Macoris ML, Galvani KC, Andrighetti MT, Wanderley DM. Assessing the effects of temperature on dengue transmission. Epidemiol Infect. 2009:137:1179-87.

53. Xu L, Stige LC, Chan KS, Zhou J, Yang J, Sang S, et al. Climate variation drives dengue dynamics. Proc Natl Acad Sci USA. 2017;1 14:113-8.

54. Shen JC, Luo L, Li L, Jing QL, Ou CQ, Yang ZC, et al. The impacts of mosquito density and meteorological factors on dengue fever epidemics in Guangzhou, China, 2006-2014: a time-series analysis. Biomed Environ Sci. 2015;28:321-9

55. Xiao FZ, Zhang Y, Deng YQ, He S, Xie HG, Zhou XN, et al. The effect of temperature on the extrinsic incubation period and infection rate of dengue virus serotype 2 infection in Aedes albopictus. Arch Virol. 2014;159:3053-7.

56. Colón-González FJ, Fezzi C, Lake IR, Hunter PR. The effects of weather and climate change on dengue. PLoS Negl Trop Dis. 2013;7:e2503.

57. Fan JH, Wei WX, Bai ZG, Fan CL, Li SL, Liu QY, et al. A systematic review and meta-analysis of dengue risk with temperature change. Int J Environ Res Public Health. 2014;12:1-15

58. Raharimalala FN, Ravaomanarivo LH, Ravelonandro P, Rafarasoa LS, Zouache K, Tran-Van V, et al. Biogeography of the two major arbovirus mosquito vectors, Aedes aegypti and Aedes albopictus (Diptera, Culicidae), in Madagascar. Parasit Vectors. 2012;5:56.

59. Thavara U, Tawatsin A, Chansang C, Kong-ngamsuk W, Paosriwong S, Boon-Long J, et al. Larval occurrence, oviposition behavior and biting activity of potential mosquito vectors of dengue on Samui Island, Thailand. J Vector Ecol. 2001;26:172-80.

60. Lima-Camara TN. Activity patterns of Aedes aegypti and Aedes albopictus (Diptera Culicidae) under natural and artifcial conditions. Oecologia Australis. 2010;14:737-44.

61. Hartberg WK. Observations on the mating behaviour of Aedes aegypti in nature. Bull World Health Organ. 1971;45:847-50.

62. Jaenson TG. Attraction to mammals of male mosquitoes with special reference to Aedes diantaeus in Sweden. J Am Mosq Control Assoc. $1985 ; 1: 195-8$

\section{Publisher's Note}

Springer Nature remains neutral with regard to jurisdictional claims in published maps and institutional affiliations.

Ready to submit your research? Choose BMC and benefit from:

- fast, convenient online submission

- thorough peer review by experienced researchers in your field

- rapid publication on acceptance

- support for research data, including large and complex data types

- gold Open Access which fosters wider collaboration and increased citations

- maximum visibility for your research: over 100M website views per year

At BMC, research is always in progress.

Learn more biomedcentral.com/submissions 